\title{
La aplicación de estrategias virtuales en la evaluación de competencias
}

\section{The application of virtual strategies in the evaluation of competences}

Recibido 21/04/2021

\author{
Zoel Isauro García Reyes \\ Maestría en Docencia Universitaria \\ Universidad de San Carlos de Guatemala \\ zoelreyes11@gmail.com \\ https://orcid.org/0000-0002-0113-7813
}

Aceptado 02/07/2021

\section{Referencia}

García Reyes, Z. I. (2021). La aplicación de estrategias virtuales en la evaluación de competencias. Revista Docencia Universitaria, 2(2), 20-31. https://doi.org/10.46954/revistadusac.v2i2.28

\section{Resumen}

La modalidad virtual en el proceso de enseñanza aprendizaje plantea muchas exigencias hacia el docente tales como: implementación de estrategias de enseñanza aprendizaje, la priorización de lo que aprenderán los alumnos, y los mecanismos para lograr ese aprendizaje. De igual manera la evaluación de las competencias es un desafío. Ante esta realidad surge como objetivo de investigación Analizar si las estrategias de evaluación virtual aplicadas por los docentes permiten evaluar las competencias alcanzadas por los estudiantes. La investigación se desarrolló desde un enfoque de investigación Mixta, con un diseño concurrente, ya que permitió conformar o corroborar resultados y efectuar validación cruzada entre datos cuantitativos y cualitativos, tenía un alcance descriptivo en la investigación obteniendo resultados más específicos sobre las estrategias virtuales y evaluación 
de competencias. La muestra estuvo integrada por 8 docentes y 194 estudiantes con quienes se trabajaron entrevistas, encuestas y un grupo focal.

Entre los resultados obtenidos en la investigación se considera que, si es efectiva la evaluación por competencias siempre y cuando se implementen nuevas metodologías y estrategias virtuales adaptables al contexto del estudiante. Desde otro punto se determina que se encuentran distintas dificultades en la aplicación de herramientas digitales, estrategias de aprendizaje virtuales para la evaluación de competencias en una educación de modalidad virtual. En el estudio se concluye afirmando y señalando la relevancia de contar con experiencias significativas que ayudaron a estudiar, comprender y desarrollar nuevas iniciativas, la necesidad de innovar los procesos de evaluación de competencias.

\section{Abstract}

The virtual modality in the teaching-learning process places many demands on the teacher such as: implementation of teaching-learning strategies, the prioritization of what the students will learn, and the mechanisms to achieve this learning. Similarly, the assessment of competencies is a challenge. Faced with this reality, the research objective arises to analyze whether the virtual assessment strategies applied by teachers allow evaluating the skills achieved by students.

The research was developed from a Mixed research approach, with a concurrent design, since it allowed to conform or corroborate results and carry out cross-validation between quantitative and qualitative data, it had a descriptive scope in the research obtaining more specific results on virtual strategies and evaluation competitions. The sample consisted of 8 teachers and 194 students with whom interviews, surveys and a focus group were worked.

Among the results obtained in the research, it is considered that, if the evaluation by competences is effective as long as new methodologies and virtual strategies adaptable to the context of the student are implemented. From another point, it is determined that there are different difficulties in the application of digital tools, virtual learning strategies for the evaluation of competences in a virtual modality education.
Palabras clave:

Estrategias virtuales, evaluación de competencia, efectividad, aprendizaje. 
Keywords:

Virtual strategies, competence evaluation, effectiveness, learning.
The study concludes by affirming and pointing out the relevance of having significant experiences that helped to study, understand and develop new initiatives, the need to innovate the competency assessment processes.

\section{Introducción}

La pandemia de COVID-19 amenazó con deteriorar aún más los resultados educativos, la pandemia tuvo un enorme impacto en la educación con el cierre de las universidades en casi todos los países del mundo, siguiendo las normativas de las autoridades correspondientes, la Universidad de San Carlos de Guatemala y sus sedes, se vio en la necesidad de suspender clases presenciales y poder adaptarse a clases virtuales, la misma fue novedad, lo que causó dificultades tanto para los docentes como para los estudiantes.

El cambio del sistema educativo de la modalidad presencial a lo virtual, constituye dos procesos muy distintos en su forma de trabajo y principalmente en la forma de evaluación, por lo que generó grandes retos y desafíos para su adaptación a las nuevas necesidades en lo que se tenía que implementar nuevos sistemas pedagógicos en la aplicación de estrategias virtuales, metodología, la forma de acceder a la realización de la evaluación, adquirir y diferenciar información y conocimiento, los medios utilizados para aplicar la evaluación de los procesos de aprendizaje que tendrían que ejercitar los estudiantes y los mecanismos adecuados para lograr sus competencias.

Los docentes continuaron el proceso educativo en la modalidad virtual tratando de hacer lo mejor posible su trabajo docente aplicando sus conocimientos adquiridos, en lo cual no se tenía una preparación para enfrentar dicha situación, las evaluaciones las realizaban conforme sus conocimientos, alcances y dominios de la tecnología, ya que en lo presencial se evaluaba por medio de pruebas objetivas o algunas otras estrategias de evaluación en donde el docente tenía el control de observar mientras el estudiante realizaba su evaluación sin que pudiera copiar o utilizar algún material de apoyo para responder a la misma, en la evaluación virtual, lo anterior no es posible, ya que se conoce al estudiante por sus aportaciones que lo evalúan frecuentemente, por lo tanto tiene que prevalecer una confiabilidad, mediación pedagógica e innovación de estrategias virtuales y adaptarlas a la evaluación de competencias para que tenga una efectividad. 
La presente investigación ha tenido como finalidad llevar a cabo un proceso por el cual se buscó la respuesta a la problemática de una forma sistemática teniendo la necesidad de conocer y explicar los diferentes factores que intervinieron en la misma, las estrategias virtuales y evaluación de competencias en los estudiantes universitarios, fue una preocupación en gran medida identificada tanto en el quehacer de los docentes, donde involucró diferentes perspectivas: la evaluación desde el punto de vista del alumno, las competencias de los estudiantes desde el punto de vista de los docente, las estrategias virtuales y acciones evaluativas desde su identificación de contenidos conceptuales, procedimentales y actitudinales.

Se abordó en la investigación el objetivo de analizar si las estrategias de evaluación virtual aplicadas por los docentes permiten evaluar las competencias alcanzadas por los estudiantes, la evaluación por competencias conduce a la dirección efectiva del proceso de enseñanza aprendizaje y a la determinación de su grado de eficiencia, se reflexiona acerca de algunas tendencias actuales en las prácticas evaluativas, lo que ha traído consigo la aparición de ideas y propuestas diversas: evaluación cualitativa, alternativa, auténtica y orientada al aprendizaje, que la sitúan en una perspectiva comprehensiva en cuanto a su objeto, funciones, metodología y técnicas, como necesidad de nuevos enfoques para la aplicación de la evaluación de competencias.

La evaluación ha de hacer más consciente y capaces a los estudiantes de cuál es su nivel de competencia, de cómo resuelven las tareas y de qué puntos son potencializados y que puntos deficientes se tenían que corregir para enfrentarse a situaciones de aprendizajes y así la evaluación fuera efectiva en la formación de los aprendizajes y se aplicaran los saberes de la educación.

\section{Materiales y métodos}

¿De qué manera las estrategias de evaluación virtual aplicadas por los docentes permiten evaluar las competencias alcanzadas por los estudiantes universitarios? La presente investigación se trabajó y se desarrolló en una línea de investigación de evaluación educativa con un enfoque mixto, buscando analizar, describir, explicar, confirmar y proponer las estrategias virtuales y evaluación de competencias para una efectividad según su aplicación. 
El diseño de investigación fue concurrente en la fase cuantitativa se centró en el diseño no experimental y en la fase cualitativa se enfocó en lo fenomenológico y se pretendió tener un alcance descriptivo en la investigación. Para estudiar la opinión de cada uno de los docentes y estudiantes, se tomó como base diferentes instrumentos de investigación tales como: la encuesta, entrevista, grupo focal y guía de observación, con la finalidad de poder obtener una buena información a profundidad y pudiera enriquecer dicho estudio.

En la investigación se utilizó dos tipos de muestras las cuales son: Muestreo probalístico en cuanto a los datos cuantitativos. Muestreo no probalístico utilizado para los datos cualitativos. La muestra se conformó por participantes voluntarios: 8 docentes que formaron parte para responder una entrevista, así también 194 estudiantes quienes respondieron la encuesta, se seleccionaron a 6 estudiantes para realizar un grupo focal y obtener información con un criterio más amplio según sus experiencias dentro del contexto educativo, además se aplicó una guía de observación para verificar diferentes indicadores dentro de la plataforma utilizada por los docentes y estudiantes.

\section{Resultados}

Los resultados de la investigación reflejaron que la aplicación de las estrategias de evaluación de manera virtual de los docentes para evaluar las competencias alcanzadas por los estudiantes fueron las siguientes: cuestionarios, videos, ensayos, análisis, evaluación oral, pruebas objetivas, trabajo interactivo y reflexivo, infografía, estudio de casos, mapas mentales, PNI, foros, organizadores gráficos, portafolios, mapas conceptuales, presentaciones, resúmenes. Las herramientas digitales que utilizaron los docentes $\mathrm{y}$ estudiantes se pudieron determinar las siguientes: plataforma de la USAC, zoom, linoit, Word, Publisher, correos electrónicos, PowerPoint, WhatsApp, canva.

En los datos cuantitativos se determinó que el $71.6 \%$ de estudiantes indican que los instrumentos o actividades con la que evalúan sus docentes es la prueba objetiva, el 51.5\%mapas conceptuales, el $63.9 \%$ ensayos, el $54.1 \%$ proyectos, el $73.7 \%$ cuestionarios, el $26.3 \%$ comprobaciones de lecturas, el $58.2 \%$ resumen, el $55.7 \%$ mapas mentales, el $2.6 \%$ otros instrumentos, el $0.5 \%$ análisis, el 0.5 murales en línea, el $0.5 \%$ forma oral y el $0.5 \%$ solo con pregunta. 
Los datos cuantitativos muestran que al $47 \%$ de estudiantes respondieron que la herramienta digital utilizada por el docente para evaluar es Google Drive, el 37\% plataforma virtual, el 8\% zoom, el 8\% plataforma de la USAC y el $1 \%$ utiliza WhatsApp. El $89 \%$ de estudiantes respondieron que las herramientas digitales utilizadas por sus docentes permiten evaluar las competencias alcanzadas y al $11 \%$ respondió que no.

La percepción de docentes y estudiantes de la efectividad de las estrategias de evaluación por competencias en entornos virtuales a raíz del COVID-19, lo describen que es satisfactoria y muy buena, siempre y cuando se aplique de la mejor manera las estrategias virtuales en la evaluación de competencias lo que permite tener una innovación, los que se tiene que tener una planificación adaptada a la modalidad virtual, ya que permite evidenciar el nivel de aprendizaje de los estudiantes; los estudiantes son más competitivos, se puede dar a conocer diferentes habilidades de los estudiantes, se puede calificar diferentes criterios utilizando diferentes instrumentos de evaluación.

También se obtuvieron aspectos muy puntuales que afectaron el proceso de evaluación tales como: el estudiante copie y pegue información o copie respuestas de la evaluación, se tiene una comodidad de los estudiantes; no hay interés de parte de algunos estudiantes, poco tiempo y saturación de actividades, hay incumplimiento de entrega de tareas, falta de discusión y profundidad de explicación de instrucciones, se dio el trabajo bajo presión, el factor económico, la falla de conectividad del internet, la adaptación a la modalidad virtual.

Los resultados cuantitativos muestran que el $52.6 \%$ de estudiantes respondieron que bueno es la valoración que merece a la evaluación de sus propios aprendizajes en la educación virtual, al 30.9\% excelente, $16 \%$ regular y el $0.5 \%$ malo. El $47.4 \%$ de estudiantes respondieron bastante de acuerdo que los resultados de sus evaluaciones han sido satisfactorios, al 38.1\% están totalmente de acuerdo, 26\% están parcialmente en desacuerdo y al $23.5 \%$ están en desacuerdo.

El 56.7\% de estudiantes de respondieron que la poca comunicación es una de las principales desventajas de la evaluación por competencias en entornos virtuales, el 32\% poca reflexión, el $18.6 \%$ carencia de capacidad crítica, el $25.8 \%$ poca responsabilidad y disciplina, el $38.7 \%$ 
falta de habilidades tecnológicas y digitales, el $8.8 \%$ falta de empatía, el $19.1 \%$ falta de automotivación, $0.5 \%$ no se cuenta con buena señal de internet, $0.5 \%$ realizar el trabajo sin dedicación y el $1.5 \%$ aprender a mejorar los medios virtuales.

\section{Discusión}

Uno de los hallazgos encontrados es la aplicación de insuficientes y recurrentes de las estrategias virtuales y herramientas digitales que se utilizan para evaluar por competencias el proceso de aprendizaje de los estudiantes, ya que las evaluaciones realizadas de manera virtual no permiten medir a su totalidad el alcance de su aprendizaje, no se puede estimular el conocimiento de los estudiantes, se dan varias oportunidades en la plataforma para realizar la misma prueba y al dar varias oportunidades se obtiene la calificación completa y sin tener mayor conocimiento.

Dicho hallazgo se relaciona directamente con la investigación de Cabrera (2007) las actividades de evaluación se tendría que centrar en una variedad de aplicación de estrategias virtuales, que permita promover el aprendizaje, así también poder medir el proceso de aprendizaje de los estudiantes y a la vez emitir un juicio crítico constructivo para su mejora que permita tomar conciencia del nivel de aprendizaje que se encuentra el estudiante según las competencias establecidas en las actividades asignadas y diseñadas sean las más apropiadas paras su realización y su formación académica. También se tiene que considerar el perfil de los estudiantes, los conocimientos, motivación, competencias, expectativas, intereses, necesidades, para obtener una evaluación que tenga una buena efectividad.

La experiencia dice que ya no se trata sólo de promover el aprendizaje y demostrarlo, en relación a lo que el estudiante tiene conocimiento sobre un tema, sino que, además, ha de ser capaz de saber demostrar, aplicar, hacer, actuar en función y en relación a lo que sabe (saber que, saber hacer y saber ser). Para que los docentes mejoren la aplicación de nuevas estrategias virtuales y la evaluación de competencias es necesario una actualización docente para tener más conocimientos sobre metodologías activas que conlleven a una evaluación efectiva. 
Sin embargo, los estudiantes afirmaron que las herramientas digitales son poco aplicadas debido al dominio de la tecnología ya que ha generado nuevos retos y desafíos para los docentes, frente a los estudiantes que poseen muchas habilidades para la utilización de estos instrumentos de alta tecnología en el proceso educativo.

Se detectó y se hace referencia también al sistema de evaluación tradicional supone apartar al alumno de cualquier fuente de información durante el tiempo que dura un examen. Esto es imposible en la educación virtual, porque los estudiantes pueden rendir el examen acompañado de todo el material que desee e, incluso, evidenciar sus respuestas con un grupo de compañeros en tiempo real, por lo tanto, no se logra tener una efectividad en el proceso de evaluación, ya que se encuentran con diferentes dificultades desde la perspectiva del docente como del estudiante.

Dicho hallazgo se puede relacionar con la investigación de Bordas (2017) hoy en día se centra la evaluación de competencias en los aprendizajes de los estudiantes y en los resultados obtenidos de una manera satisfactoria y puede desarrollar habilidades competitivas en cada uno de los alumnos, la incidencia de las estrategias virtuales en la evaluación de competencias tiene que incidir lo que es la planificación docente, las actividades cooperativas, reflexivas, así también la formativa.

La experiencia dice que cuando se comprende la esencia de evaluar con evidencias a lo largo del proceso, podrá aumentar su eficacia para educar por competencias, creciendo como docente e investigador, así también cuando una evaluación está bien diseñada, la calificación refleja un aprendizaje significativo de la competencia, y el hecho de trabajar por obtener una calificación y trabajar por aprender.

Otro de los hallazgos es que el $70 \%$ de conocimiento adquirido se refleja en las respuestas de la evaluación, siendo este bastante satisfactorio para el alumno, el $20 \%$ presenta comodidad o desinterés en la verificación del aprendizaje adquirido. El $10 \%$ solo desea llegar a la zona mínima para seguir estudiando.

Con la experiencia de los estudiantes dan a conocer que las pruebas se realizan de manera autónoma e independiente si son factibles ya que se miden las competencias establecidas en la evaluación, pero si se 
pide copia o se hace uso de distintas herramientas que hacen el trabajo por el estudiante, no se logra aprender nada.

Se identifica otro hallazgo, no existe efectividad en la forma de evaluación, por lo tanto, el grado de eficiencia o nivel es mínimo para que el estudiante pueda demostrar como evidencia su desempeño. Se considera que es un aprendizaje con un nivel intermedio porque solo se evalúa teóricamente y no se sabe si los alumnos después de seguir los procesos lograron el nivel de competencias que se requería.

Por lo tanto, algunos docentes indican que, si es efectiva la evaluación por competencias siempre y cuando se apliquen nuevas metodologías adaptables al contexto de los estudiantes, y otros docentes dicen que no es efectiva ya que se encuentran con diferentes factores tecnológicos y pedagógicos que limitan mucho para poder alcanzar las competencias de los estudiantes en su proceso de aprendizaje.

La evaluación por competencias en la educación virtual, se adquieren las formas prácticas para desarrollar métodos efectivos de evaluación y calificación a través de aspectos a calificar, donde lo efectivo sea lograr las evidencias imprescindibles para tener el dominio de la competencia con un esfuerzo razonable, en el tiempo previsto y con los recursos disponibles, siendo esas evidencias ciertamente significativas de la competencia evaluada.

Se puede decir según ANECA (2013) la evaluación de competencias se puede considerar como un proceso de recopilación de diferentes evidencias que permitan evidenciar los resultados de aprendizaje del estudiante según las competencias establecidas y así mismo su dominio, las estrategias virtuales deben estar alineadas a la evaluación de competencias y pensar en qué herramientas serán las más apropiadas para determinar el grado en el que el aprendizaje ha sido alcanzado por el estudiante, teniendo en cuenta el tiempo y los recursos disponibles para su utilización en su proceso de enseñanza aprendizaje.

Algunas conclusiones y recomendaciones obtenidas en la investigación son las siguientes: las estrategias virtuales aplicadas por los docentes en la evaluación de competencias fueron las siguientes: cuestionarios, videos, ensayos, análisis, evaluación oral, pruebas objetivas infografías, mapas mentales, exposiciones virtuales, y las herramientas digitales 
utilizadas por los docentes y estudiantes universitarios se pueden mencionar: plataforma de la universidad, Word, Publisher, correos electrónicos, WhatsApp, drive, zoom, camba, y por ende la forma de evaluar las competencias de los estudiantes se realizaron con trabajos interactivos y reflexivos, rubrica, lista de cotejo, portafolios, a través de los logros alcanzados por los estudiantes, por lo tanto durante el proceso de enseñanza aprendizaje no se generó un mayor porcentaje de efectividad de la evaluación de competencias en el entorno virtual, se tuvieron distintas dificultades en la aplicación de estrategias virtuales, se siguió utilizando las pruebas objetivas, poca frecuencia de uso de la plataforma de parte de los estudiantes, no se tenía innovación en aplicación de técnicas, herramientas, actividades, para variar y renovar la evolución de competencia y así poder generar un aprendizaje efectivo en los estudiantes.

La efectividad de las estrategias de evaluación de competencias en entorno virtual a raíz del COVID-19, dadas las complejidades de la evaluación de competencias no se tenía ningún modelo o diseño que pudiera ser utilizado en la aplicación de las acciones y procedimientos para evidenciar sus procesos y logros de aprendizaje, lo que fue un reto y un desafío enfrentar dicha situación, ya que los docentes y estudiantes se fueron adaptando a la educación virtual con sus conocimientos, teniendo una respuesta a la continuidad de los procesos de enseñanza aprendizaje, lo que influyó el nivel de competencias digitales que poseía cada docente y estudiante, y sobre todo la percepción que tiene la utilidad de las mismas como la herramienta de mediación pedagógica, problemas de conectividad, acceso a las tecnologías y acomodamiento.

Es importante y útil mejorar la efectividad de la evaluación de competencias en la educación virtual, centrándola en la aplicación de los tres tipos de contenidos, conceptuales, procedimentales, actitudinales, que identificarán a los estudiantes a través de sus saberes, hechos, conceptos, habilidades, actitudes, en el desarrollo de las diferentes actividades que permitan que el docente utilice distintos elementos al momento de trabajar con los alumnos para conseguir las capacidades expresadas en las competencias, así mismo hacer uso de la propuesta de estrategias virtuales para aplicarlas en la evaluación de competencias y así generar un aprendizaje efectivo, motivador e innovador en los estudiantes.

Es recomendable potenciar las estrategias virtuales en la evaluación de competencias para lograr una efectividad, donde los docentes y 
estudiantes sean los actores principales de las prácticas y acciones evaluativas en espacios dialógicos y priorizados sobre cómo llevar a cabo el análisis y reflexión de los aprendizajes que se desean lograr a través de las competencias establecidas, así mismo se debe visualizar el enfoque evaluativo por competencias centrado en lo formativo brindando una oportunidad de desarrollo de aprendizajes que refuerzan y establecen los procesos de autoaprendizaje y la autorregulación de las practicas pedagogías en la adaptación de estrategias virtuales para la evaluación de competencias, que permitirá fomentar la responsabilidad, crítica y autocrítica de los propios estudiantes para que mejoren sus procesos de aprendizaje, esto indica la necesidad de variar e innovar las prácticas y las formas de evaluar para implementar diseños y prácticas orientadas a lo formativo para lograr tener una evaluación de competencias muy efectiva y un aprendizaje significativo.

\section{Agradecimientos}

Se le brinda un cordial agradecimiento a la Dra. Walda Paola Flores Luin, docente del curso de Tesis, así mismo al Asesor: M.A. Roberto Antonio Martínez Palma, por contribuir y permitir recurrir a sus valiosas capacidades y conocimientos científicos para obtener la orientación para culminar todos los procesos antes mencionados y hacerlos de la mejor manera, logrando resultados satisfactorios.

\section{Referencias}

ANECA. (Marzo de 2013). Guía para la redacción, puesta en práctica y evaluación de los resultados del aprendizaje. ENQA.

Bordas, M. I. (2017). Estrategias de Evaluaciòn de los aprendizajes centrados en el proceso. EDUCREA.

Cabrera. (2007). La Evaluación de Competencias en un Entorno Virtual de Aprendizaje. . Unidad de Apoyo al EEES de la UOC, 8.

\section{Góngora, M. M. (2017). EVALUACIÒN DE COMPETENCIAS TÈCNICAS INFORMALES. SEMI, 14.}


Facultad de Humanidades Escuela de Estudios de Postgrado

\section{Sobre el autor}

\section{Es Licenciado en Pedagogía y Administración Educativa por la Universidad de San Carlos de Guatemala.}

\section{Copyright (c) Zoel Isauro García Reyes}

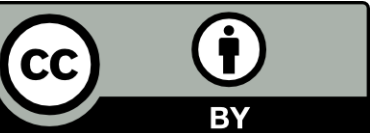

Este texto está protegido por una licencia Creative Commons 4.0.

Usted es libre para compartir, copiar y redistribuir el material en cualquier medio o formato y adaptar el documento, remezclar, transformar y crear a partir del material para cualquier propósito, incluso comercialmente, siempre que cumpla la condición de atribución: usted debe reconocer el crédito de una obra de manera adecuada, proporcionar un enlace a la licencia, e indicar si se han realizado cambios. Puede hacerlo en cualquier forma razonable, pero no de forma tal que sugiera que tiene el apoyo del licenciante o lo recibe por el uso que hace. 